\title{
Transatlantica
}

Revue d'études américaines. American Studies Journal

2 | 2014

Aesthetics of Theory in the Modern Era and Beyond / Photographie documentaire

\section{Pictures versus Pictures}

Exposition Robert Mapplethorpe - Grand Palais (Paris), 26 mars - 13

juillet 2014

\section{Jonathan Maho}

\section{(2) OpenEdition}

\section{Journals}

Electronic version

URL: https://journals.openedition.org/transatlantica/7035

DOI: $10.4000 /$ transatlantica.7035

ISSN: $1765-2766$

\section{Publisher}

Association française d'Etudes Américaines (AFEA)

Electronic reference

Jonathan Maho, "Pictures versus Pictures", Transatlantica [Online], 2 | 2014, Online since 05 March

2015, connection on 06 February 2023. URL: http://journals.openedition.org/transatlantica/7035 ; DOI: https://doi.org/10.4000/transatlantica.7035

This text was automatically generated on 6 February 2023.

\section{cc) (1) 8}

Creative Commons - Attribution-NonCommercial-NoDerivatives 4.0 International - CC BY-NC-ND 4.0

https://creativecommons.org/licenses/by-nc-nd/4.0/ 


\title{
Pictures versus Pictures
}

\author{
Exposition Robert Mapplethorpe - Grand Palais (Paris), 26 mars - 13 \\ juillet 2014
}

\author{
Jonathan Maho
}

1 La rétrospective Robert Mapplethorpe (1946-1989) présentée dans la galerie sud-est du Grand Palais se voulait être la première exposition muséale consacrée en France à sa photographie. En insistant sur l'aspect inédit d'une présentation dans «les galeries nobles " ' d'un musée, le commissaire, Jérôme Neutres, rappelait le traitement généralement réservé à son œuvre dans les institutions présentant uniquement les tirages d'exposition produits par la Mapplethorpe Foundation. La sélection proposée au Grand Palais se voulait exhaustive, à la fois du point de vue du type ou du contenu des œuvres. Ainsi, bien que Mapplethorpe soit connu pour ses images explicitement sexuelles, les expositions qui lui ont été consacrées ont eu jusqu'ici tendance à mettre en valeur l'aspect formel de sa photographie et à exclure les images les plus choquantes $^{2}$. Tout l'enjeu d'une exposition Mapplethorpe en 2014 était donc bien d'offrir une nouvelle lecture de sa photographie.

2 Si sur les quelque 4000 œuvres produites par l'artiste entre 1968 et 1989 seulement la moitié est constituée de tirages argentiques noir et blanc, ceux-ci représentent malgré tout l'écrasante majorité des travaux exposés depuis sa disparition. J. Neutres attribue le caractère incomplet des expositions monographiques proposées jusqu'alors notamment aux conditions d'exposition spécifiques nécessaires à la présentation des pièces uniques, des travaux devenus fragiles et précieux, comme c'est le cas du portrait d'Andy Warhol montré à Paris et devenu en 2006 l'une des œuvres les plus chères de l'histoire de la photographie ${ }^{3}$. Le paradoxe, concernant l'un des photographes américains les plus célèbres, ne se résume donc pas seulement à sa relative absence dans les programmes des institutions muséales françaises mais correspond également à la méconnaissance de son travail, bien plus riche que ce qu'ont laissé supposer la plupart des expositions monographiques proposées jusqu'à aujourd'hui.

3 C'est cette richesse que souhaitait donc mettre en valeur le commissaire de l'exposition du Grand Palais. "Photographe plasticien ", tel était le rôle attribué à Mapplethorpe dans la quadrilogie programmée par la Réunion des Musées Nationaux: après 
l'exposition d'un photographe de mode (Helmut Newton), d'un reporter (Raymond Depardon), et avant celle consacrée à un portraitiste (Seydou Keïta), la rétrospective Mapplethorpe venait compléter un cycle sur la photographie qui visait, selon J. Neutres, à situer le médium dans l'histoire de l'art et à « l'institutionnaliser » dans les galeries nationales ${ }^{4}$. Une sélection de 263 travaux permettait ainsi de montrer l'ambition manifeste du photographe new-yorkais mais également de souligner une grande diversité des matériaux et techniques employés au cours de sa carrière.

Cette variété d'approche était mise en avant dès le début de l'exposition avec certains de ses derniers travaux. Des tirages platine, des pièces uniques encadrées par l'artiste, des sculptures (judicieusement disposées entre les tirages argentiques): l'œuvre produite à la fin de sa vie était, si l'on ne considère que les techniques utilisées, relativement diversifiée. En revanche, à cette période, la palette de ses motifs de prédilection est assez limitée : il n'est alors plus question que de portraits, de nus ou de photographies de sculptures.

5 En proposant une chronologie inversée de son parcours, le commissaire en avait donc défini à Paris une lecture à rebours, permettant dès l'introduction de mettre l'accent sur cette diversité insoupçonnée. Ce parti-pris offrait de multiples avantages: commencer par la mort de l'artiste (du SIDA à l'âge de 42 ans) et donc, mettre en avant l'aspect tragique de sa vie, permettait de refléter l'aspect théâtral de sa photographie. L'histoire racontée par cette mise en scène était également celle d'un photographe certes libéré des contraintes financières (il avait dû attendre longtemps pour réaliser des tirages platine, très coûteux) mais sachant également sa fin proche: on peut voir dans sa désaffection pour le nu, au profit de photographies de sculptures, un désintérêt croissant pour la chair à mesure que son état de santé s'aggravait. L'exposition s'ouvrait ainsi avec son dernier autoportrait, celui d'un homme malade disparaissant dans l'ombre derrière le pommeau d'une canne en forme de tête de mort. On ne peut également s'empêcher de penser que le parcours chronologiquement inversé visait à mettre en évidence l'aspect formel de son travail dans la mesure où cette exposition était avant tout destinée à participer à «l'institutionnalisation » de la photographie au Grand Palais. Par ailleurs, puisqu'il ne produisit aucune œuvre à caractère sexuel à cette époque, l'introduction pouvait ainsi être soft, suffisamment en tout cas pour ne pas choquer d'emblée le public du Grand Palais. Alors que «cock» était plus loin traduit correctement sur les cartels et les cimaises, le même mot cité en français dans une citation de l'artiste se limitait à « le sexe » dans la première salle de l'exposition...

6 Tout en étant fidèle aux intentions de l'artiste et, comme le dit dans le catalogue de l'exposition le président de la Réunion des Musées Nationaux Jean-Paul Cluzel, sans laisser «la décence commune » définir ce qui devait être présenté dans l'exposition, l'habile préambule proposé par le commissaire avait donc le mérite d'être juste à la fois d'un point de vue didactique (grâce à une introduction séduisante) et historique (il n'y a plus de sexe dans l'œuvre de Mapplethorpe à partir de 1985). L'exposition, tout comme les articles lui étant consacrés dans la presse, s'appliquaient également à souligner la volonté de l'artiste d'être reconnu comme sculpteur, utilisant certes un appareil photo mais travaillant avec la lumière comme d'autres travaillent le marbre. Ses photographies telles que celles de la série des Thomas ou Ajitto démontraient à Paris sa volonté d'exposer des corps sculpturaux, mais elles révèlent également une fine connaissance de l'histoire de l'art dans laquelle s'inscrivait concrètement la pratique de Mapplethorpe. L'artiste prenant par exemple la relève d'un d'Hippolyte Flandrin ou 
d'un George Dureau en jouant sur des analogies : ainsi pour Ajitto, le modèle pose pour Mapplethorpe comme il posa pour Dureau, tel le Jeune Homme Nu Assis au Bord de la Mer peint par le peintre français au XIX ${ }^{e}$ siècle. Les sculptures disséminées dans la première moitié de l'exposition ainsi que les pièces uniques - des photographies avec passepartout en soie, encadrés par l'artiste lui-même - permettaient de pousser la démonstration encore plus loin : certaines œuvres de Mapplethorpe s'inscrivent dans l'histoire de la photographie en rappelant les toutes premières images de l'histoire, elles-mêmes devenues des objets grâce aux indissociables des écrins de cuirs et de velours dans lesquels elles étaient présentées. Il faut sans doute ici rappeler que Mapplethorpe collectionnait les daguerréotypes depuis $1971^{5}$.

7 La précision (des tirages) et la perfection (des formes) étaient ainsi mises en valeur dans la première partie de la rétrospective. Avec une mise en scène rappelant les variations de Fred Holland Day sur la figure du christ au Salon de Philadelphie en 1899, un superbe accrochage attendait le visiteur à mi-parcours et mettait en lumière l'influence de l'iconographie religieuse dans l'œuvre de Mapplethorpe. Face à des tirages couleurs (splendides, eux aussi), ce mur comprenant des photographies, une sculpture en forme de croix et une petite installation à l'allure de chapelle miniature, évoquait également le loft new-yorkais de Mapplethorpe dont les murs étaient recouverts des œuvres qu'il collectionnait. Cette présentation très esthétique était enfin à Paris un moyen de rendre hommage aux expositions conçues par l'artiste qui, on le sait, portait lui-même beaucoup d'attention à l'accrochage.

8 Mapplethorpe proposait très souvent des présentations groupées de ses photographies. Elles pouvaient être aussi alignées sur une même grille et encadrées de la même manière suivant un procédé plus sommaire et également retenu au Grand Palais pour la présentation de ses Polaroids. Malheureusement l'accrochage, aussi pertinent qu'il ait été d'un point de vue esthétique, mettait également en évidence les limites du concept de l'exposition.

9 Celle-ci se terminait par les débuts de Mapplethorpe et une sélection de 47 instantanés alignés sur un même mur. Très différentes des accrochages de type "salon » (avec un mur-tableau composé d'images aux formats et techniques variés), ce type de présentation était utilisée par le photographe afin de parvenir à une appréciation égale de photographies considérées comme faisant partie d'un ensemble indivisible. Une image de fleur ou d'un pénis en érection pouvait ainsi chez Mapplethorpe côtoyer un paysage et un portrait d'enfant ; l'installation X, Y, Z Portfolios (1978-1981), constituée de trois rangées de treize photographies allant du SM aux nus en passant par les natures mortes, en est le meilleur exemple. Or cette œuvre, qui est à la fois manifeste et exposition miniature (Mapplethorpe définit lui-même la façon de la présenter), n'a pas été montrée au Grand Palais. Et malgré son importance dans la carrière de l'artiste, on en vient presque à comprendre son absence, qu'elle ait été ou non volontaire. En effet au Grand Palais, natures mortes, images SM et nus ne pouvaient être présentés ensemble.

10 Ainsi, sur le mur des Polaroids, pas une seule photographie à caractère sexuel. Pour qui connait l'œuvre de Mapplethorpe cela ne peut paraitre qu'incongru puisque l'instantané accompagna l'artiste dans sa découverte du sexe. En effet, après avoir d'abord utilisé pour ses œuvres des photographies trouvées dans des magazines, il s'était découvert photographe au moment où il s'acceptait homosexuel; les expérimentations, qu'elles aient été sexuelles ou photographiques, étaient 
intrinsèquement liées. Ce qui signifie que parmi les 1621 Polaroids parvenus jusqu'à nous aujourd'hui, de très nombreux sont explicitement sexuels. Au Grand Palais, les plus malicieux feront remarquer que l'on voyait bien ici un godemichet (dans un lavabo), là Peter Berlin en tenue «bondage ». Soit. Mais n'en déplaise à J-P. Cluzel, qui dans l'introduction du catalogue insistait sur la présence des images les moins consensuelles, ces dernières étaient bel et bien exclues de la sélection de Polaroids présentée à Paris. Un oubli ?

11 Non. Au Grand Palais le sexe était rangé ailleurs. Dans une salle exiguë, interdite aux mineurs, était regroupé une vingtaine de travaux jugés trop crus pour être présentés dans le reste de l'exposition. Cette pièce à l'entrée dissimulée par un rideau avait tout du cabinet de curiosité, reléguant symboliquement des pratiques et des œuvres très complexes (et parmi les meilleures chez Mapplethorpe) au rang de bizarrerie. Mapplethorpe n'a jamais proposé ce type d'artifice, non sans raison. Bien au contraire : si ces photographies devaient être présentées dans la même exposition, alors elles étaient rassemblées par l'artiste sur les mêmes murs, tous motifs confondus, comme cela fut le cas au Whitney Museum en $1988^{6}$. Dans la salle classée X du Grand Palais étaient donc isolées des pièces uniques, certaines réalisées très tôt (à partir de photos de magazine, découpées puis recouvertes de peinture), d'autres plus tard, par exemple à la fin des années 1970, lorsque sa photographie ne s'était pas encore fortement formalisée. Parmi ces œuvres, certaines des plus pertinentes chez Mapplethorpe: l'artiste posant là, retourné vers l'objectif, tenant dans sa main un long fouet noir (courant sur le sol et introduit dans son rectum) comme d'autres tiennent le déclencheur à distance d'un appareil photo. Mapplethorpe en pleine maitrise de son art devient également maitre de l'expérience du spectateur, un spectateur dont le malaise face au regard accusateur du photographe est nécessaire à l'accomplissement de l'expérience entre dominé et dominant. Même ailleurs, face à son propre regard dans les miroirs intégrés aux cadres par l'artiste, il est impossible pour le visiteur d'échapper à cette confrontation... à moins de se tenir à l'écart, c'est-à-dire de ne pas pénétrer dans la pièce classée $\mathrm{X}$.

12 Chez Mapplethorpe, l'expérience de l'œuvre, aussi difficile puisse-t-elle être, devait recréer chez le spectateur " $a$ feeling in the stomach $»^{7}$ correspondant à sa première expérience de la pornographie. Isoler les photos les plus osées revient donc à limiter cette expérience, empêchant le spectateur de lâcher prise face à une œuvre esthétisant toutes choses, qu'il soit question d'une fleur ou d'un pénis. On trouvait bien deux photographies de sexes en (semi) érection dans le reste de la rétrospective parisienne mais malheureusement réduits, à la faveur d'une analogie, à leur statut d'organes inoffensifs encadrés par des fleurs aux pistils protubérants. Figure imposée depuis deux décennies dans toutes les expositions sur Mapplethorpe ("montrons un pénis mais, de grâce, seulement si l'on peut le voir comme une fleur »), cette comparaison réduit l'œuvre à sa plus simple expression (esthétisante, s'entend). Mapplethorpe avait certes dit qu'il photographiait une fleur ou un pénis de la même manière, mais il n'a jamais dit qu'il s'agissait de la même chose. Quant à cette esthétique si particulière, elle n'était pas pour lui une fin en soi, mais bien un moyen de « tout » montrer, de forcer le spectateur à tout apprécier de la même façon. Si l'on examine les $X, Y, Z$ Portfolios, installation où tout est présenté sur le même plan, il paraît évident que le cloisonnement, ou la définition de catégories, est bien vain pour Mapplethorpe. 
13 dans le catalogue par la satisfaction du président de la Réunion des Musées Nationaux relative la présentation des fameuses photographies les moins consensuelles, il devient difficile de ne pas remarquer la relative absence de sexe au Grand Palais, rendue d'autant plus évidente que le mot «cock» était présent partout (des citations aux cartels, en passant par les textes du catalogue d'exposition). Aussi, pourquoi insister pendant la moitié de l'exposition sur l'intérêt que portait Mapplethorpe à la sculpture ${ }^{8}$ (et son influence sur sa pratique de la photographie) pour reléguer le sexe, qui l'intéressait bien plus que le marbre, à l'arrière-plan? Avec pour fâcheuse conséquence sa disparition du mur de polaroids. Alors que dans les années 1970, pour Mapplethorpe, Polaroid = Sexe, en 2014 l'équation semble être devenue impossible, en conséquence de quoi se trouve éclipsée, «l'unité organique » caractéristique de l'œuvre, évoquée par exemple par le critique d'art Arthur Danto'. Même l'idée originale de chronologie inversée se trouvait mise à mal par l'artifice de la salle isolée, puisque se retrouvaient à la fin de l'exposition parisienne des œuvres produites dans les années 1980 (tel Cock, datant de 1985).

Plus que d'être conscient, en 2014, de devoir « tout » montrer, il importe de prendre la mesure d'une présentation débarrassée d'artifices sûrement accommodants pour le commissaire mais n'aidant pas à comprendre ce qui est original dans sa pratique de la photographie. Pourquoi ne pas avoir tout simplement interdit l'exposition au moins de 18 ans $?^{10} \mathrm{Et}$ ainsi permettre à l'œuvre de Mapplethorpe d'être appréciée comme l'artiste l'avait toujours souhaité ? Peut-être aurait-il fallu faire davantage confiance aux visiteurs. Ou à l'artiste. L'exposition du Grand Palais semble finalement avoir souffert d'une trop grande attention portée à l'expérience du spectateur, modérée à l'excès jusque dans la scénographie. Ainsi la lumière douce, les couleurs sobres et la moquette si épaisse qu'elle étouffait le bruit des pas, relevaient-elles d'une mise en scène étudiée pour être des plus engageantes. Mais l'extrême soin apporté à l'ambiance créée tout particulièrement pour l'exposition paraît avoir trop compté, certains choix dans la présentation de l'œuvre ayant été définis par des impératifs parfois contraires à une meilleure compréhension de sa photographie. Une photographie qui, rappelons-le, se suffit à elle-même lorsqu'il s'agit de séduire le spectateur. Cette rétrospective invite au passage à se pencher sur l'importance grandissante des scénographies dans la préparation des expositions, celle du Grand Palais illustrant très bien les limites de l'exercice.

15 Les difficultés liées à l'exposition du travail de Mapplethorpe ne datent pas d'hier. Paradoxalement, c'est avec deux photographies rappelant cette problématique que débutait l'exposition du Grand Palais. Intitulées toutes les deux Pictures, ces deux images montrent la main du même l'artiste, écrivant le même mot mais habillé de façons différentes. À gauche Mapplethorpe porte une belle montre et une chemise, à droite, un gant et un bracelet de cuir. Ces deux images étaient présentées en 1977 sur les deux faces d'un même carton d'invitation correspondant à deux expositions organisées conjointement, l'une étant présentée chez la galeriste Holly Solomon, l'autre au centre d'art The Kitchen, à New York. Inutile de préciser que le contenu des deux expositions était radicalement différent. L'invitation annonçait donc bien plus que l'exposition : elle rendait compte au premier chef d'un dualisme assumé se reflétant dans sa photographie, voire, dans le personnage lui-même - un photographe aussi bien à l'aise Uptown qu'à SoHo. Du reste, pour l'artiste pour qui «une exposition ne 
commence pas lorsque vous entrez dans une galerie " mais bien «à la minute où vous recevez l'invitation par la poste $»^{11}$, le carton conditionnait la réception de son œuvre avant même le vernissage. Ce que l'invitation suggérait donc également, c'était que la tenue ou le contexte pouvaient bien changer, son art, lui, restait le même. Qu'importe le lieu d'exposition, qu'il y soit question de sexe ou de fleurs, il ne s'agissait dans les deux cas que de photographies. Et nous devrions être capables de les voir comme telles.

Peut-être pouvons-nous voir dans cette difficulté persistante une confirmation, s'il en était besoin, de la pertinence de sa photographie, non pas pensée pour être belle ou choquante, mais jouant sur différents registres (esthétiques et psychologiques) pour nous confronter à nos propres limites. Que l'on soit adepte du SM ou novice. Visiteur ou commissaire d'exposition. D'ailleurs, il ne faudra pas attendre de la prochaine rétrospective Mapplethorpe une approche différente : en 2016 à Los Angeles, le Getty Museum et le LACMA (qui ont acquit conjointement les archives de la Mapplethorpe Foundation en 2011) présenteront une rétrospective en deux parties, l'une dédiée à sa pratique formaliste de la photographie, l'autre insistant sur son côté «bad boy». Deux concepts d'exposition mettant en valeur la dualité dans l'œuvre de Mapplethorpe, entre libération et contraintes. En 2016, ce sera donc Dionysos versus Apollon... « Pictures » versus « Pictures », encore une fois.

\section{BIBLIOGRAPHY}

DANTO, Arthur C., Playing with the edge : the photographic achievement of Robert Mapplethorpe, Berkeley, University of California Press, 1996

MORRISROE, Patricia, Mapplethorpe : a biography, New York, Random House, 1995

NEUTRES, Jérôme (dir.), Robert Mapplethorpe, Paris, Réunion des musées nationaux - Grand Palais, 2014

Mapplethorpe - Rodin, Arles / Paris, Actes Sud / Musée Rodin, coll. « Beaux Livres », 2014

\section{NOTES}

1. Interview du commissaire par Valérie Duponchelle, « Mapplethorpe au Grand Palais : "Le Jean Genet de la photo" ", Le Figaro, rubrique Culture, 26 mars 2014, http://www.lefigaro.fr/culture/ 2014/03/26/03004-20140326ARTFIG00152-mapplethorpe-au-grand-palais-le-jean-genet-de-laphoto.php

2. La présentation de l'œuvre de Robert Mapplethorpe peut être jugée problématique à plusieurs égards. Premièrement en raison de son contenu, puisque le sexe est très présent dans sa photographie; l'artiste a par ailleurs consacré certaines de ses séries aux pratiques SM. Mais l'exposition de son travail est également jugée difficile en raison de sa réception dans le contexte des batailles culturelles aux USA : opposant conservateurs et libéraux, elles ont influencé la lecture faite de sa photographie depuis que son exposition a été au centre d'une polémique retentissante à la fin des années 1980. En réaction, il est souvent proposé des expositions 
consacrées à ses images les plus sages (Kunsthalle Dusseldorf 1992; Guggenheim New York $2004 ; \ldots)$.

3. Andy Warhol, 1987 (tirage platine sur toile, cadre unique et panneaux de soie), vendu par Christie's \$ 643.200 à New York en octobre 2006.

4. Valérie Duponchelle, art. cit.

5. Certains des Daguerréotypes collectionnés par l'artiste sont accessibles à Los Angeles au Getty Research Institut, où sont conservées les archives léguées par la Mapplethorpe Fondation.

6. « Robert Mapplethorpe », Whitney Museum of American Art, New York, 28/07-23/10/1988

7. Mapplethorpe cité par Patricia Morrisroe dans Mapplethorpe : a biography, Londres, Papermac, 1995, 11

8. Ce que proposait également une exposition montrée parallèlement au musée Rodin en comparant l'œuvre de deux artistes («Mapplethorpe Rodin», Musée Rodin, Paris, 08/04-21/09/2014).

9. Arthur C. Danto, Playing with the edge: the photographic achievement of Robert Mapplethorpe, Berkeley, University of California Press, 1996, 10

10. Option retenue pour la présentation en 2013 des $X, Y, Z$ Portfolios au LACMA à Los Angeles. http://www.lacma.org/art/exhibition/robert-mapplethorpe-xyz

11. Patricia Morrisroe citant l'artiste dans Mapplethorpe : a biography, op. cit., 1995, 128-9 